\title{
CONTABILIDAD PARA LA EQUIDAD Y LA INCLUSIÓN SOCIAL: PROPUESTAS PARA UNA INVESTIGACIÓN INTERDISCIPLINARIA A LARGO PLAZO*
}

\author{
GABRIEL RUEDA DELGADO**
}

PONTIFICIA UNIVERSIDAD JAVERIANA

Recibido/ Received/ Recebido: 15/02/2011 - Aceptado/ Accepted/Aprovado: 01/05/2011

\begin{abstract}
Resumen
Colombia transita hacia la convergencia internacional, hacia un conjunto único de prácticas de reconocido valor técnico y aceptación a nivel mundial. Sin embargo, lejos de contribuir a la solución de problemas de la estructura económica y social del país, la adopción de dichas prácticas beneficiaría solo a algunos agentes contribuyendo a mantener invisibles, las necesidades de otros grupos sociales no necesariamente vinculados al mercado. El presente documento prepone adelantar actividades de investigación de largo aliento, que, a partir de reconocer los alcances y condiciones de la contabilidad, acuda a otras disciplinas para el estudio de alternativas teóricas y aplicadas para aportar soluciones a la aguda situación social y económica por la que atraviesa el país. El documento aporta diagnósticos y plantea alternativas conceptuales y metodológicas que inviten a la comunidad contable del país a hacer aportes para comprender y explicar el papel que la contabilidad puede jugar en nuestro entorno nacional sin desconocer los procesos de globalización.
\end{abstract}

Palabras clave: Convergencia contable, contabilidad social, neoliberalismo, acción comunicativa, equidad, inclusión.

\section{ACCOUNTING FOR EQUITY AND SOCIAL INCLUSION: PROPOSAL FOR A LONG TERM INTERDISCIPLINARY RESEARCH}

\begin{abstract}
Colombia is passing through an international convergence to a unique practice group of acknowledged technical value and acceptance worldwide. However, far from contributing to a problem solution of Colombian economic and social structure, the implementation of such practices would benefit just a few agents contributing to maintain invisible the needs of other social groups not involved with the market. This paper propose to advance on long term research activities, which, from accountability approaches and conditions, look to other disciplines to study theoretical and applied alternatives with the purpose of contributing with solutions for social and economic situation of the country. This paper subscribes diagnosis and establishes conceptual and methodological alternatives to understand and explain the role that accountability can play in our national scope considering globalization processes.

Keywords: Accountable convergence, social accountability, neoliberalism, communicative action, social equity, social inclusion.

El presente trabajo hace parte del proyecto de Tesis doctoral Contabilidad para la equidad y la inclusión, presentada y aprobada por el Comité Doctoral del Doctorado en Ciencias Sociales y Humanas de la Pontificia Universidad Javeriana, en diciembre de 2010. Contador público de la Universidad Nacional de Colombia, docente de la Pontificia Universidad Javeriana de Bogotá (Colombia). Correo electrónico: gabriel.rueda@javeriana.edu.co
\end{abstract}




\title{
CONTABILIDADE PARA A EQUIDADE E A INCLUSÃO SOCIAL: PROPOSTAS PARA UMA PESQUISA INTERDISCIPLINAR A LONGO PRAZO
}

\begin{abstract}
Resumo
Colômbia visa convergir em um único conjunto de práticas internacionais de reconhecido valor técnico e aceitação mundial. No entanto, a adoção de tais práticas não contribuiria para a solução de problemas da estrutura social e econômica do país, senão que beneficiaria apenas alguns agentes e ajudaria a manter invisíveis as necessidades de outros grupos sociais não necessariamente ligados ao mercado. Este artigo propõe levar a cabo pesquisas a longo prazo que, além de reconhecer as possibilidades e as condições da contabilidade, entre em contato com outras disciplinas para o estudo de alternativas teóricas y aplicadas para fornecer soluções à situação social $e$ econômica aguda que enfrenta o país. O documento apresenta diagnósticos e propõe alternativas conceituais e metodológicas para convidar a comunidade de contabilidade do país para contribuir a entender e explicar o papel da contabilidade no ambiente doméstico, sem ignorar os processos de globalização.
\end{abstract}

Palavras chave: Convergência contável, contabilidade social, neoliberalismo, ação comunicativa, equidade social, inclusão social.

Rueda, G. (2010) Contabilidad para la equidad y la inclusión social. Propuestas para una investigación interdisciplinaria a largo plazo En: Revista de la Facultad de Ciencias Económicas de la Universidad Militar Nueva Granada. rev.fac.cienc.econ, XIX (1).

JEL: M401, M41, M49.

\section{Introducción}

La condición de disciplina social de la contabilidad implica, el estar atento a los desarrollos, cambios y necesidades del entorno que no solo provienen del mercado y las empresas sino de la sociedad en su conjunto. Es menester moral de cada disciplina y de cada investigador, cuestionar, en este caso a la contabilidad, sobre el alcance social de su conocimiento y ponerlo al servicio de la sociedad en conjunto.

Históricamente la contabilidad parece tener un papel instrumental en la aplicación de políticas económicas y empresariales y un rol inexistente cuando las mismas políticas incluyen asuntos sociales. Por ello, el presente documento pretende plantear opciones y alternativas para hacer investigación contable, pero de carácter interdisciplinario, para abordar como objeto de estudio la equidad y la inclusión social. En esa medida el presente documento antes que ofrecer respuestas, plantea preguntas, se apoya en posibles referentes teóricos y propone alternativas metodológicas para abordar el cuestionamiento central que se plantea.

El texto plantea en primer lugar un contexto en el que se pretende ubicar el rol que actualmente se le está dando a la contabilidad, y en particular en los más recientes avances de regulación económica, analizando de forma crítica dicho rol frente a las realidades sociales que vive el país. De dicho contexto se formula una pregunta central que se pretende sirva de guía para la construcción de una investigación de largo plazo, y que posteriormente se apoya en algunos referentes conceptuales y teóricos que puedan ser útiles para abordar el estudio y comprensión de la pregunta formulada. Finalmente se realiza una propuesta de cómo poder abordar el estudio de lo planteado. 
En todo caso, siendo una propuesta para crear un proceso de investigación de largo plazo, ninguna de estas secciones deben ser consideras como recolecciones exhaustivas, referentes únicos o caminos excluyentes para abordar el abordaje de lo que aquí se propone.

\section{Contexto}

En el mes de julio de 2009, se promulga en Colombia la Ley 1314 conocida como la Ley de Convergencia contable $e^{1}$, cuyo artículo 1 , de los objetivos de la Ley señala que (resaltado está fuero del texto original):

- “(...) es una manera de intervención económi$c a$, en particular regulando la manera como las empresas deben presentan sus estados financieros.

- (...) para mejorar la productividad, la competitividad y el desarrollo armónico de la actividad empresarial de las personas naturales y jurídicas, nacionales o extranjeras (...)

- (...) con el propósito de apoyar la internacionalización de las relaciones económicas, la acción del Estado se dirigirá hacia la convergencia de tales normas de contabilidad, de información financiera y de aseguramiento de la información, con estándares internacionales de aceptación mundial, con las mejores prácticas y con la rápida evolución de los negocios".

Tres temas fundamentales, para los alcances de la investigación que se propone, se destacan de esta sección de la Ley que han sido resaltados en negrilla. En primer lugar, la contabilidad y la regulación para la presentación de información financiera, es una manera como el Estado hace intervención económica en las empresas, lo cual evidencia la relación entre economía, regulación y contabilidad. En segundo lugar, se asume en la Ley que la contabilidad mejora la gestión empresarial y, en esa medida, es un instrumento útil para la consecución de los objetivos de las organizaciones y en tercer lugar, que las prácticas contables que se adoptan son una estrategia para la internacionalización de la economía.

Esta articulación de visiones económicas, empresariales e internacionales, corresponde exactamente con las propuestas que el Instituto de Economía Internacional realiza, en el sentido de promover la necesidad:

"De reformas institucionales domésticas con implicaciones para la estabilidad de los mercados financieros internacionales, que se extienden a las prácticas de auditoría y contabilidad reconocidas en todo el planeta, de modo que los prestamistas puedan evaluar con precisión las condiciones financieras de los bancos y las empresas a las que van a hacer préstamos" (Eichengreen, 2000, 13).

En ese marco el papel del Fondo Monetario Internacional es,

“... estimular que las normas que impulsen organismos como la Junta internacional de estándares contables IASB (por sus siglas en inglés) sean adoptadas y cumplidas por los países y otorgando préstamos a condición que se sigan ciertos pasos".

Sin duda, los contenidos de la Ley Colombiana corresponden en muy buena medida con estas "políticas globales".

1 Por la cual se regulan los principios y normas de contabilidad e información financiera y de aseguramiento de información aceptados en Colombia, se señalan las autoridades competentes, el procedimiento para su expedición y se determinan las entidades responsables de vigilar su cumplimiento La ley, que es la manera como el Gobierno Colombiano define el papel de la contabilidad dentro de las estrategias de inserción de la economía nacional en la lógica económica, productiva y financiera mundial y de paso le permite "poner al día" la regulación en dicha materia respecto de lo que ya la mayoría de países ha hecho dentro del proceso de estandarización contable mundial denominado Convergencia y que se lidera desde la International Accounting Standard Board IASB organización de carácter privado. 
En consecuencia, los países adoptan este tipo de regulaciones en un marco mundial de Nueva Arquitectura Financiera Internacional NAFI, de tal forma que los gobiernos colaboren con este tipo de disposiciones que favorecen, mantienen y amplían la liberalización de los mercados financieros, la cual es vista, desde estas opciones, no solo como positiva para la distribución eficiente de la inversión, la movilización del ahorro, la nivelación del consumo y la diversificación de la cartera, sino además como inevitable e irreversible.

Las prácticas estandarizadas de presentación de información empresarial, buscan reducir las asimetrías de acceso y uso de la información que los mercados de capital poseen, impidiendo correcciones bruscas y crisis, y limitando que los inversionistas y prestamistas que no estén bien informados, hagan inferencias a partir de las acciones de otros y reaccionen en consecuencia (Eichengreen, 2000, 3).

Hoy día, las prácticas mundiales de contabilidad se desarrollan, por medio de acuerdos entre reguladores como el mundial IASB y el norteamericano FASB (Financial Accounting Standar Board, también privado) hacia un conjunto de estándares de presentación de reportes financieros y estados contables (IASB-FASB, 2010, 10) que permitan tomar decisiones adecuadas a los inversionistas (actuales y potenciales) y prestamistas financieros (actuales y potenciales) a partir de la entrega de información útil para decisiones de inversión y financiación (Tua 2000, 116) con instrumentos clásicos y nuevos en mercados de capitales (valores y financieros).

El proceso de estandarización de la contabilidad global beneficia a dichos usuarios (inversionistas y prestamistas) no solo con la reducción de costos en la preparación y consolidación de la información, al elaborarse toda bajo una sola base de contabilidad, sino fundamentalmente por medio de la reducción de los riesgos para la inversión y el otorgamiento de préstamos en la medida que la información estandarizada aumenta su comprensibilidad para dichos usuarios.

Esta orientación en materia de contabilidad financiera, contribuye a la libre circulación del capital multinacional, lo cual está directamente relacionado con la política económica del país, en el sentido de que esta última busca favorecer y atraer la inversión extranjera generando regulación que, como la contable, le sea conveniente, como parte de una estrategia general de "desarrollo". En los últimos años, desde el 2003, en América Latina y en Colombia (Castillo 2010, 100), hemos visto un incremento sustancial de presencia de Empresas Multinacionales en prácticamente todos los sectores de la economía para las cuales, unas prácticas contables que usen una base diferente a los IFRS (International Financial Reporting Standard, emitidos por el ya mencionado IASB) no les resulta funcional para sus intereses.

El incremento de la inversión extranjera directa conlleva además a la implementación de los modelos de negocio de las grandes empresas mundiales que llegan al país. Las empresas multinacionales hacen su presencia en el país aprovechando las condiciones favorables que encuentran para la reducción de costos, por vías como la flexibilización laboral, incentivos fiscales, etc. La Contabilidad se convierte en el puente que comunica al capital aportado (o prestado) que "viaja por todo el mundo", con la administración de las empresas que utiliza dichos recursos y los asigna con el fin de maximizar la retribución a dicho capital. Por esta razón la información contable que se genera mide, valora, reconoce y divulga lo que es pertinente para dichos usuarios, en esta forma de capitalismo financiero y para cumplir los requerimientos de la NAFI.

La regulación contable resulta así ligada a intereses que son el reflejo de condiciones de poder de ciertos grupos que lo detentan, en especial los inversionistas y prestamistas financieros multinacionales agentes fundamentales en los modelos actuales del capitalismo (Halabi 2004, 33). Dicho de otra manera, la internacionalización que la contabilidad estandarizada permite, es una ligada con el modelo empresarial neoliberal (Rueda, 2011a) implementado en Colombia desde la década de los 90 o antes (Uribe, 2010; González, 2002, 89), que favorece la deslocalización, incrementa la utilidad de la organización, apoyada en regulaciones nacionales que colaboran con el orden internacional. 
Pero no toda esta dimensión de lo internacional puede explicarse desde el modelo económico, que parece impuesto "desde afuera". Los grupos económicos y empresariales nacionales y las elites del país por razones diversas (Stallings, 1994, 70) se sienten identificados y alineados con esta dimensión de la internacionalización. La convergencia contable no presenta mayores resistencias entre el empresariado Colombiano y al contrario, este parece haberlo apoyado en las discusiones previas a la promulgación de la Ley. El modelo contable se implementa con el liderazgo del gobierno nacional, en medio de coincidencias entre actores internacionales y grupos económicos nacionales, y sus consecuentes representantes políticos.

Los vínculos entre regulación contable en Colombia y referentes internacionales no son nuevos. Estas prácticas mundiales venían influyendo y determinando los marcos normativos desde hace varios años (Consejo Técnico de la Contaduría Pública, 2006, 43; Mantilla, 2002). Por ejemplo con el Decreto 2649 de 1993 que regulaba la práctica de la contabilidad en Colombia. Sin embargo, la "similitud" conceptual se iba desdibujando con la existencia de normas técnicas diversas, múltiples reguladores, y en general visiones diversas del papel de la información contable y su regulación.

Pese a estas "similitudes" en el año 2003, el Informe del Banco Mundial conocido como el ROSC ${ }^{2}$ identificó que Colombia era aún de los pocos países que no habían modificado su marco normativo para hacerlo consistente con el internacional, en un proceso de alcance mundial conocido como convergencia. En el año 2008, se inicia un nuevo intento normativo que bajo la coordinación del Ministerio de Comercio Exterior, condujeron a la promulgación de la Ley 1314 de 2009, que adopta prácticas mundiales de contabilidad, auditoría etc. Dicha Ley, bajo lo anteriormente expuesto, es la disposición gubernamental a acoger los lineamientos que la NAFI impone, respetando los lineamientos de estabilidad financiera mundial.
Sin embargo, es evidente que en la economía Colombiana, tras varios décadas de estas formas de inserción internacional y de políticas y modelos económicos, la gestión empresarial está lejos de contribuir a la construcción de una sociedad más equitativa, desarrollada, justa e incluso digna para la mayoría de la población, como lo evidencias las altas cifras de pobreza e indigencia producidas por el gobierno nacional y estudios de la CEPAL sobre concentración de ingreso en nuestro país.

En la búsqueda de la eficiencia, la competitividad y el mercado, se ha generado un esquema de reducción de costos permitido y alentado por normas de flexibilidad laboral, reducción de la tarifa de impuestos y erosiones de las bases gravables, maquilización de empresas nacionales (en su mayoría pyme) limitando la formación de capital nacional e impidiendo una acumulación para el desarrollo económico y social generalizado, aumentando los ya de por si profundos desequilibrios entre departamentos y regiones.

Al mismo tiempo, y con una fuerte relación con estas lógicas económicas, la pobreza, la inequidad, la violencia, el desplazamiento forzado (Ibáñez, 2006), el desempleo rural y urbano, el creciente nivel de informalidad laboral, la precaria calidad de los servicios de salud, educación y justicia, pese al aumento del gasto público en dichos rubros, (Núñez, 2009), la extrema concentración de tierra y riqueza (Machado, 2006), hacen que los cambios estructurales económicos, sociales e incluso políticos que requiere el país para su desarrollo sean mucho más urgentes y apremiantes.

Igualmente, la inversión extranjera sigue aumentando en el país, impulsando en algunos sectores el crecimiento pero al mismo tiempo Colombia sigue siendo de los países con mayores niveles de concentración de la riqueza, con desmejoramiento en los niveles de empleo formal, aumento de la informalidad y disminuciones marginales de la pobreza y la

2 Reportes sobre la Observancia de Estándares y Códigos, que fue realizado por una misión en Colombia durante varios meses del 2003 y que en este caso evaluaba la cercanía de las prácticas, normas e instituciones contables nacionales, respecto de las mundiales. 
indigencia. Esto supone la insuficiencia de la estrategia para generar desarrollo.

Pese a la posibilidad de complementariedad entre mercado y Estado (Messner, 1999, 77), los enfoques de desarrollo en la mayor parte de América Latina y en particular en Colombia, se han centrado en favorecer los intereses privados, en defensa de la inversión extranjera sin que exista un aparato empresarial competitivo. Visto así la adopción contable no conduce a cambios fundamentales en la estructura del país y por tanto el estudio de modelos alternativos al neoliberalismo y a la forma de internacionalización por la vía de la Inversión Extranjera Directa, son parte fundamental en el desarrollo de este trabajo de investigación.

Estas condiciones para el desarrollo no solo tienen efectos económicos sino incluso políticos y en particular en asuntos tan importante como el ejercicio de la democracia (Rueda, 2011b) que la han venido circunscribiendo a una serie de procedimientos electorales, que terminan conformando cuadros de gobierno local regional y nacional que actúan sin tener de fondo una decisión política para transformar el estado de cosas en el país y de legislar a favor de la mayoría de la población. En este entendimiento de democracia, las empresas privadas adquieren una visión de "ciudadanos corporativos" aunque desprovistas de muchas de la obligaciones y deberes que dicho entendimiento implica, entre ellas el generar información para el debate público, en una sociedad como la Colombiana.

El Legislativo, al hacer regulación como la mencionada Ley 1314 de 2009, sigue actuando para atender necesidades de actores multinacionales cada vez más globales como parte de los propósitos que el neoliberalismo persigue, mientras que otros actores $y$ agentes sociales se siguen invisibilizando en las prácticas de revelación y divulgación de información contable de las empresas. En este sentido es necesario que la contabilidad contribuya a develar las relaciones inequitativas que se dan entre los actores que confluyen en la gestión empresarial y al mismo tiempo hagan visible las relaciones que se dan entre empresa y actores sociales que terminan invisibilizadas en la contabilidad pensada para los inversionistas y prestamistas.

La contabilidad estandarizada y la información que ella produce, no es una para la alteridad y la construcción de una sociedad incluyente. Su mensaje es puramente instrumental y usado solo desde las visiones economicistas de la realidad empresarial, pese a reconocer que la Empresa interactúa con la sociedad y distintos agentes económicos de muchas formas complejas.

La Información que la contabilidad puede dar de la acción empresarial debe ser capaz de reconocer al otro para generar, en espacio público, la construcción de una sociedad distinta, más equitativa $e$ incluyente, por medio de criterios de valoración, medición y revelación de información que trasciendan las necesidades de inversores y prestamistas. Una internacionalización distinta, pensada no solo para la inversión extranjera (sin excluirla o estigmatizarla), que se apoye en esquemas de desarrollo nacional renovadores y que ayude a una mayor interacción a nivel intra regional y regional como estrategia para insertarse en el mundo, requiere de una nueva responsabilidad de la gestión empresarial y por consiguiente de una nueva forma de contabilidad que trascienda la estandarización impulsada por la ley.

Las transformaciones deben buscarse en medio de las resistencias y la búsqueda de cambios a las desigualdades en intercambios, y se traduce en luchas contra la exclusión, la inclusión subalterna, la dependencia, la desintegración y la descalificación (De Souza, 2003, 209). Una contabilidad desde abajo y contra hegemónica (en el sentido de De Souza, 2003, 171) para la internacionalización de la economía en condiciones de equidad e inclusión. Cómo lograrlo es el objeto central en la propuesta de configuración del presente propuesta de investigación a largo plazo.

Una contabilidad que genere diálogo y deliberación social y devele los intereses y efectos que el modelo actual produce. Una contabilidad que no esté solo al servicio del capital multinacional ni de la lógica neoliberal que empobrece y marginaliza a cada vez más 
nacionales. Una contabilidad para la emancipación (De Souza, 1999) y no para la dominación. Información para la interpretación social, e incluyente de los demás agentes que interactúan en el medio empresarial.

De esta manera es necesario preguntarse como centro de la investigación que se propone ¿de qué forma la información contable que las empresas generen, puede contribuir a "dar cuenta" de los efectos sociales de un nuevo acuerdo social, propicio para una internacionalización y desarrollo económico más equitativo e incluyente en el país $y$ en esa medida retroalimentarlo y ayudar a su fortalecimiento?

Se hace necesario, que la información empresarial, (es decir la que se incluyen en los estados financieros y aquella que la complementa y ayuda a explicarla como las notas a los estados contables e informes de gestión de la gerencia) contribuyan a la construcción de una sociedad más justa, equitativa y en últimas democrática. De esta forma, la responsabilidad social empresarial deja de ser una estrategia comercial y publicitaria y se convierte en una herramienta poderosa para la construcción de un proyecto de nación diferente por medio de una gestión empresarial más incluyente y digna, soportada en información contable social y no solo financiera. El trabajo en esa medida pretende, en el largo plazo y desde una aproximación conceptual, ejemplificar como puede ayudar la contabilidad a la sociedad, desde información cuantitativa y cualitativa y que sirvan de punto de partida para una relación teórica y práctica entre contabilidad y equidad e inclusión.

Una contabilidad que devele los efectos sociales de la gestión empresarial y permita, desde ejemplos concretos de organizaciones reales, aumentar y cualificar el debate público. Así la contabilidad aporta información para nuevas compresiones de la realidad social y económica del país, y genera interpretaciones $y$ visiones que puedan ser usadas para tomar decisiones en la construcción de un nuevo concepto de país y, de una democracia que soporte el crecimiento económico, pero que cada vez beneficie a más colombianos.

\section{Referentes teóricos a ser considerados}

Esta propuesta toma como punto de partida que la información contable empresarial debe ser concebida como un bien público (así sea de una empresa privada) que mejora las condiciones de deliberación social sobre el modelo económico, la inserción internacional de la economía y la gestión empresarial en beneficio de múltiples actores sociales. Lo anterior implica apoyarse en varios referentes conceptuales y disciplinas (Sierra 2010, 5) para buscar tentativas soluciones a la pregunta planteada enmarcadas en un propuesta de investigación a largo plazo.

Lo dicho anteriormente requiere (sin detrimento de un marco teórico conceptual más elaborado y más rico que debe resultar del propio proceso de investigación a realizar en la investigación) una serie de premisas o apuestas teóricas, disciplinares e incluso políticas, que se identifican con las tradiciones de las teorías críticas, en especial las aplicaciones de ella a las relaciones internacionales, y la teoría de la acción comunicativa y su aplicación en la contabilidad. Al mismo tiempo con una visión de lo económico centrada en la distribución, en la equidad y la inclusión; y una re significación de la responsabilidad social empresarial y del papel que juegan en ello la contabilidad como disciplina y de la información que ella produce que aporta en la construcción de alternativas para una sociedad diferente a la que actualmente vivimos.

La creación de propuesta formula una apuesta por relacionar la contabilidad, con la democracia (Habermas, 2006, 2009) (e incluso la emancipación en los términos de De Souza, 2006a, 2006b) al menos en tres dimensiones. Una primera forma es aportando información, derivada de la gestión empresarial, que politice el debate y en particular ponga en la palestra pública para la deliberación social cualificada, la estrategia de inserción económica del país en lo internacional y el modelo social de producción imperante en los esquemas como el neo liberalismo. Lo anterior supone dejar de entender a la contabilidad solo como el resultante de una serie de condiciones económicas y regulativas previas, y la convierte en un insumo que ayude a pre formar la realidad. 
Una segunda manera de relación tiene que ver con el aumento y cualificación de la deliberación públi$c a$, que por la politización anterior se genera. Esto implica, por ejemplo, que la información contable estandarizada debe ser cuestionada en términos de quien la genera, para qué lo hace y como espera ese alguien que sea interpretada dicha información. El cumplir con los estándares de Contabilidad, pretende darle a la información contable (debidamente auditada) criterios de validez de dicha información depotenciando su uso como fuente para consensos posteriores en la sociedad.

De una tercera manera, se busca fortalecer el concepto de empresa y en particular el de la responsabilidad social de dicho actor. En este sentido, una contabilidad que politiza el modelo social de producción y mejora la calidad del debate público tiene repercusiones, al entender la empresa como una coalición de intereses y en particular de dos maneras concretas. La primera plantea que los intereses de las empresas, se sujetan a unos intereses mayores socialmente construidos y definidos por actores socio económicos más y mejor informados respecto de la misma gestión empresarial. La segunda significa que la responsabilidad empresarial, no es definida a voluntad del ente que emite la información que le es pertinente para mostrarse responsable ante la sociedad, sino que la regulación misma, le indica cuales son los mínimos de información que debe revelar, por ejemplo respecto de las variables de pertinencia social para la equidad y para la inclusión.

Los referentes teóricos que esta propuesta supone en consecuencia, requieren incluir de manera articulada:

- Las teorías de las relaciones internacionales y las formas alternativas de ver el mundo.

- Visiones amplias de la globalización que implique significar dicho proceso como una construcción social no hegemónica.

- Modelos de desarrollo económico distintos al neo liberalismo y por consiguiente,

- Una nueva forma de comprender el papel que la contabilidad juega en la construcción de una nueva realidad económica, social y empresa- rial, en esto caso como aplicación de la Teoría de la Acción Comunicativa.

Una contabilidad para la equidad, la inclusión social y en consecuencia para la democracia deliberativa que implique que las empresas divulgan información de pertinencia social, económica y financiera que alimenten el debate público, de una sociedad capaz de limitar el alcance del mercado, en procura de lograr unos objetivos políticos y económicos nacionales, y desde allí construir la inserción del país en el mundo.

Para comprender como estos marcos y enfoques servirán de soporte para la construcción de una investigación interdisciplinario para la contabilidad al servicio de la equidad y la inclusión social, es necesario hacer una breve descripción de los mismos.

\subsection{Relaciones internacionales y Globalización}

Las visiones de inserción de la economía nacional en el mundo y de la globalización producen problemas relevantes que se hacen concretos en las organizaciones y que la contabilidad puede ayudar a reflejar por medio de la información que revela. Así por ejemplo cuando la internacionalización es entendida como formular políticas para facilitar la llegada de inversión extranjera directa a todos los sectores de la economía, o cuando las mismas políticas parten de entender que la globalización un fenómeno homogeneizador al cual hay que "obedecer" incondicionalmente, la estandarización contable es el mejor camino para responder a estas lógicas.

Pero lo anterior, resulta problemático e insuficiente conforme a lo planteado en el contexto. Revisar el papel de la contabilidad para una inserción internacional de lo económico diferente a lo que actualmente existe y hacerlo reconociendo los procesos de globalización contemporáneos necesariamente lleva a apoyarse en referentes sobre las Relaciones Internacionales como disciplina (per se interdisciplinaria) y la globalización como objeto de estudio.

Las relaciones internacionales como campo disciplinar, han venido evolucionando en el marco de grandes debates a partir de los cuales se han gestado 
una serie de teorías para explicar y comprender esta dimensión de las ciencias sociales (Weber, 2005; García, 2000; Elias-Sutch, 2007; Griffiths, 2007; Dunne, 2007). Existe actualmente una "explosión" de teorías de las relaciones internacionales que sin embargo no han impedido una predominancia y visión hegemónica que los autores categorizan como realista para ver y entender el mundo. Los enfoques realistas, surgidos en el mundo bi polar siguen imperando y desarrollándose en las teorías liberales (y neo liberales) que plantean que el objeto de las relaciones internacionales (RI) es la construcción de instituciones del sistema internacional y el comercio mundial.

En este sentido, es importante preguntarse si el "mundo" al que hoy nos referimos cuando hablamos de "inserción" es el resultante de unas visiones particulares y predominantes. De esta forma las teorías no predominantes, pueden ser una "herramienta" conceptual poderosa para plantear alternativas al papel de la contabilidad en el "mundo actual" pero siendo este objeto de diversas miradas no hegemónicas y muy próximas a teorías constructivistas y críticas.

Un ejemplo de estas tensiones y debates entre enfoques predominantes y alternativos en las teorías de las relaciones internacionales (TRI) se ilustra con el paradigma del Estructuralismo y el subdesarrollo en relaciones internacionales (Barbé, 2007, 68), que a diferencia de los transnacionalistas, no ve en el capitalismo un factor tendiente a la integración, y a la cooperación sino la causa de los problemas del subdesarrollo existentes en el planeta, en donde la imagen del mundo es una conflictiva, expresada en términos marxistas de explotación.

"La economía y la política, a nivel interno y a nivel internacional, se integran y no pueden explicarse independientemente una de otra" afirma Roger Tooze, citado por Barbé $(2007,71)$.

Con igual pertinencia para esta propuesta de investigación, las teorías constructivistas de las Relaciones internacionales, no asumen el sistema internacional como "algo dado" sino por el contrario algo que se construye de manera intersubjetiva en donde la construcción de intereses e identidades va a determinar su centro de atención. Las preferencias de los agentes que intervienen en el escenario internacional (en clara relación con las dimensiones política y económica de cada nación) son el producto del entorno social. La identidad nacional siempre está por construir y desde ella se define la manera como se logra la inserción.

De esta manera, construir información empresarial ampliada a lo social como la que se propone trabajar en un proceso de investigación de largo aliento, puede aportar elementos para la toma de decisiones sociales en la construcción de intereses nacionales que definan la manera como debemos insertarnos, al tiempo que puede aclarar para quién lo haremos y de que forma. Sin duda, una contabilidad capaz de develar los efectos sociales que la internacionalización actual produce, ayudará a la construcción social de intereses nacional para una inserción diferente.

De la mano de las relaciones internacionales y sus teorías, la globalización surge como objeto de estudio que en buena parte determina el horizonte actual de la contabilidad como disciplina. El caso de la globalización y lo contable ha estado marcado y definido por las políticas impulsadas por organismos internacionales que siempre se han leído como ineludibles (Mantilla, 2002; Montilla et al., 2005). Al mismo tiempo, los argumentos en contra de la adopción de estándares internacionales de contabilidad (que es la mayoría de la profesión) plantean problemas de soberanía y ajustes en el mercado laboral y profesional (Martínez, 2000).

En el caso de la globalización, resulta pertinente tener presente al menos tres diversos entendimientos respectos de sus características y alcances. Se trata de las visiones hyperglobalistas (Luke, 2007; Held, 2004; Lechner, 2008), escépticas (Wallerstein, 1999; Huntington, 1993; Lechner, 2008) y transformacionistas (Castells, 1999; Giddens, 2000; Keohane, 2000). La globalización para estos últimos es híbrida, compleja y diferenciada y ha sido en la historia un proceso de avances y retrocesos, que ayuda a comprender nuevos y amplios papeles en la 
actividad económica y social, al mismo tiempo que plantean una revisión del papel del Estado frente a la información empresarial para que no sea solo de cooperación con la institucionalidad internacional.

Pueden plantearse afinidades conceptuales entre teorías de las RI predominantes y visiones hyper globalistas, y entenderlas como causas determinantes de la contabilidad global de hoy día.

\subsection{Neoliberalismo: consecuencias y alternativas}

El neoliberalismo históricamente surge de importantes cuestionamientos ideológicos, económicos, políticos e incluso culturales que el Estado de Bienestar genera para la eficiencia económica de los países (Anderson, 1996; Callinicos, 1993; Boron, 2008). Su idea fundamental en lo económico es que los precios sean determinados libremente por el mercado, bajo las leyes de oferta y demanda y por lo tanto se hace necesario que no existan "obstáculos" para que el mercado actúe (Restrepo, 2003, 19). Dentro de los principales obstáculos están los costos laborales, la excesiva carga fiscal, y en general la regulación que impida el desarrollo de la iniciativa empresarial privada. A nivel mundial, el modelo neo liberal ha impulsado una visión financiera del capital y su acumulación (en lugar de comercial o industrial), y ha generado un cambio en el esquema empresarial más descentralizado, des localizado y a la vez más global (Ahumada, 2006, 29; Harvey, 2008). Al mismo tiempo ha reducido el alcance de la regulación y del papel del estado, para hacer del mercado el principal orientador de la economía y de la sociedad.

Como proyecto político y social, el neoliberalismo requiere, entre otras cosas, para poder mejorar las utilidades y maximizar la acumulación de capital, un creciente ejercito de desempleados que abaraten el costo de la mano de obra, un sistema educativo que forme personas altamente capacitadas y competentes para actuar en medios flexibles, un sistema de desregulación que permita la libre circulación del capital financiero multinacional de inversión (Bellamy, 2007, 47; Arrighi, 2007) y una reducción de las tasas impositivas para mejorar las posibilidades de competir en mercados globalizados.
El neoliberalismo se implementa en América Latina $y$ en Colombia mediante una serie de fases que incluyen el ajuste macroeconómico, la desregulación y el crecimiento de la Inversión Extranjera Directa. Sin embargo la forma como el neoliberalismo se acoge depende en gran medida, como ya se dijo, no solo de las condiciones internacionales sino que es fuertemente condicionado por el papel de las élites económicas nacionales y por el nivel de "presión" que las multinacionales tienen sobre los Gobiernos nacionales (Stallings, 1994, 68; Ahumada, 2006; Portes, 2004; Evans, 2007; Kalmanovitz, 2007; Reviez, 2004).

El modelo (no de manera mecánica o ahistórica, sino más bien de forma compleja y como resultado de una serie de condicionantes históricos y de las estructuras sociales y económicas de cada país), al tiempo que ha sido efectivo en el control de la inflación, el incremento de las utilidades empresariales privadas, y en la reducción en el papel del Estado, ha generado precarización de empleo, aumento del sub empleo y el empleo formal y ha marginalizado y excluido a cada vez grupos poblacionales más grandes (Cesde, 2009, 151). Igualmente ha motivado a que las regulaciones fiscales beneficien a grandes empresas, por vía de múltiples y complejas exenciones, beneficios y reducciones en la tarifa de impuestos directos como el de Renta, mientras que se incluyen dentro de los contribuyentes a grupos de personas de la clase media cada vez mayores (Estrada, 2004, 104). Los gobiernos se financian cada vez con impuestos al consumo y menos con impuestos directos como la renta.

Estos y otros muchos efectos del neoliberalismo producen serios problemas sociales que la contabilidad debe abordar, informar y divulgar ampliando su alcance a más agentes y actores económicos tratados con igual relevancia por la información empresarial. De allí la importancia de hacer de la contabilidad una forma de acción comunicativa, como se expondrá más adelante, relacionada directamente con los efectos que el modelo produce y que es usado por las empresas para explicar los resultados económicos obtenidos. 


\subsection{La empresa como coalición de intereses}

El hegemónico discurso neoliberal, ha constituido a las empresas en "ciudadanos corporativos" capaces de realizar la integración social, en beneficio de los agentes que en ella intervienen.

Forrester $(1997,93)$ señala sin embargo, que se le han dado más cargas morales de las que una empres privada, en un ambiente desregulado, está dispuesta a asumir, en la medida que su interés sigue siendo maximizar el resultado económico por medio de herramientas y modelos gerenciales que producen altos costos sociales que justamente impiden la integración social. Estas cargas morales se ven expresas en la literatura sobre responsabilidad social corporativa RSC. Así por ejemplo, para Gago $(2005,4)$ y Jiménez $(2008)$ la idea principal de la responsabilidad social corporativa (RSC).

"Consiste en que las empresas tienen la obligación de trabajar para conseguir una mejora del bienestar social".

Existe hoy una importante discusión en torno a si la dirección general de la Economía debe provenir de los privados y en particular de las empresas o del estado y de cuales intereses resultan "superiores" y correctos (Habermas, 2002a, 282).

La empresa, entendida como coalición de intereses, toma recursos de la sociedad y debe procurar la maximización de esta relación de manera equilibrada y no solo la generación de valor para la propiedad. Entender a la empresa como consecuencia de una teoría de stakeholders, (Manzurul, 2006) donde los que intervienen tienen los mismos derechos y deberes, puede ayudar a potenciar el papel de la información en la construcción de un espacio público para la deliberación social en torno a la participación de dichos actores en la gestión.

Estimular la inversión privada (extranjera o nacional) y procurar la competitividad y eficiencia, no puede seguirse dando solo en beneficio de agentes (también extranjeros o nacionales) como los inversionistas y prestamistas financieros, y en detrimento sistémico de otros intervinientes como proveedores, estado, trabajadores, etc. Un concepto distinto de empresa soporta la idea de una contabilidad para la equidad y la inclusión como la expuesta a lo largo de este trabajo.

Una empresa que busca el incremento de sus utilidades de manera legítima (Rueda, 2010a), no solo dando cumplimiento a la ley sino superando su propio alcance, acompañada de un marco social, políti$\mathrm{co}$, económico, moral y cultural que la obligue (y no solo la invite) a construir bienestar puede darse de mejor manera cuando aporta información pertinente para la deliberación pública de su gestión.

\subsection{La teoría de la legitimidad y crítica en Contabilidad. Aportes para un nuevo contrato social}

Diversas perspectivas de la contabilidad pero en especial la crítica, ponen de presente que el rol de la disciplina no solo es instrumental sino que debe aportar elementos a la transformación de la sociedad (Gómez \& Ospina, 2009; Larrinaga 1999; Giraldo 2008). Se trata de plantear sobre que referentes debe apoyarse la contabilidad y bajo que perspectiva, para que, dentro de ambientes globales, aporte teorías e instrumentos para un desarrollo nacional y la inserción en la lógica económica mundial.

Diversos aportes de la Teoría crítica (Ryan, 2004; Rueda, 2010b) son retomados en el presente trabajo, entre ellos la necesidad de abordar visiones interdisciplinarias para abordar realidades complejas, el sentido social del conocimiento para la emancipación, la construcción y sentido social del espacio público (más amplio que el interés público que impulsan los organismos como la IASB) y la generación de una nueva cultura democrática, que serán referentes en el desarrollo del presente proceso de investigación.

Las realidades complejas de hoy, que la información contable puede ayudar a develar, pueden tener que ver por ejemplo con asuntos como: la participación del sector financiero en el valor agregado económico de la empresa que en muchos casos puede obtener remuneraciones mayores que la misma propiedad; antecedentes jurídicos de la tierra que las empresas usan para explotación agro industrial, 
los cuales pueden "provenir" de fallidos procesos de reforma agraria o de desplazamientos forzados a la población campesina; la forma como se dan las relaciones entre empresas y trabajo, que en la generalidad de los casos conduce a la precarización del empleo formal, el sub empleo y el desempleo estructural entre otras.

Ejemplos como los indicados ayudan a generar, cuando se hacen agregados por sectores, una nueva conciencia política entorno a la gestión empresarial, el manejo de los recursos y aumenta los niveles de responsabilidad social de las empresas, pero con Trascendencia Social como lo denomina este trabajo y su capacidad de rendición de cuentas sin que ello altere lo que estrictamente se puede considerar secretos industriales.

Es en este sentido que los aportes que brinda la Teoría de Legitimidad en Contabilidad (provenientes de la Economía Política) pueden contribuir a identificar la manera como los intereses y orientaciones estratégicas de las empresas, se puede "alinear" con intereses mayores construidos socialmente. Varios autores (Archel, Husillos, Larrinaga, Spence, 2009; Deegan, 2006) señalan la necesidad de un análisis más detallado de las relaciones entre empresa sociedad y estado, mediadas por la información contable, podrían:

"Ayudar a ampliar el poder explicativo del enfoque tradicional de la Teoría de la Legitimidad y demarcar líneas de investigación que permitan avanzar hacia una sociedad más justa y democrática que son los objetivos principales del proyecto de contabilidad social (Gray, 2002)".

Justamente el alcance de esta propuesta de investigación va en desarrollo de esas nuevas líneas de investigación en contabilidad social, soportadas en la Teoría de la Legitimidad.

\subsection{Contabilidad para la equidad y la integración social. El soporte en la teoría de la acción comunicativa}

El apartado final del marco teórico procura articular los temas anteriores en una visión de la contabilidad que supere las visiones instrumentales que se han mostrado anteriormente como limitadas para poder abordar este tipo de objetos de estudio. La propuesta de la investigación es que dicha articulación se logra al evidenciar las relaciones que existen entre información contable y la Teoría de la Acción Comunicativa, como se explica brevemente a continuación.

En un sentido amplio, se espera que desde la información divulgada se generen una serie de interacciones sociales, donde los agentes y usuarios interesados en los resultados empresariales, "converjan" en el uso de dicha información para la construcción de planes intersubjetivamente definidos (bajo una visión amplia de empresa como ya se mencionó). Dichas interacciones, (Habermas, 1990, 1985, 2002a) lingüísticamente mediadas:

"Se distinguen ante todo por el mecanismo de coordinación de la acción, y en particular según que el lenguaje natural se utilice solo como medio en que tiene lugar la transmisión de informaciones o también como fuente de integración social. En el primer caso habla de acción estratégica y en el segundo de acción comunicativa".

La información que la empresa divulga por medio de la contabilidad, para efectos de extender su alcance de manera apropiada con lo descrito en el problema de investigación, debe generar Acción Comunicativa y no solo la transmisión de información o acción estratégica o con arreglo a fines.

Lo anterior significa hacer de la información contable un medio para que el oyente y el hablante participen de procesos comunicativos en cuanto dicen algo, y comprenden lo que se dice, y tengan que adoptar una actividad realizadora o preformativa de la realidad (Habermas, 1985, 38). Visto así, la contabilidad no es consecuencia objetiva de la realidad sino aporta elementos para que los agentes $y$ actores preformen la realidad a construir intersubjetivamente. Comprender lo que se dice implica participación y no solo observación.

Cuando la información contable supera la visión de racionalidad económica Weberiana para la propie- 
dad y la acumulación de capital (Habermas, 2002b, 214) se convierte en un foro para la deliberación en donde:

"La esfera pública política, se convierte en un cuerpo de resonancia para detectar los problemas que afectan al conjunto de la sociedad y formadora de opiniones públicas" (Habermas, 2009, 141; Habermas, 2006, 285).

El modelo deliberativo de democracia, mejorado por revelaciones de información empresariales amplias, no busca, a diferencia de la democracia liberal, la agregación correcta de los intereses de los individuos, orientados al éxito sino a la racionalidad de los discursos y de la negociaciones a los cuales la contabilidad puede hacer un aporte desde la información de la gestión empresarial, que se divulga no a decisión de la misma empresa, sino a conformidad de las necesidades del debate público.

Que la información empresarial satisfaga las necesidades de usuarios para tomar decisiones de inversión y financiamiento es algo fundamental para la actividad empresarial, pero insuficiente cuando se quieren comprender $e$ interpretar, las relaciones que surgen entre empresa y sociedad. Desde este punto de vista, la visión ampliada de la contabilidad que este trabajo pretende construir es una que complementa la tradicional y funcional información estandarizada.

Desde esta apuesta de investigación, lo que en primer lugar puede aportar la contabilidad y la interpretación que ella revela, tiene que ver con develar la manera como los resultados empresariales, que dicha información refleja, se explican y comprenden en el marco del modelo neoliberal, del esquema de internacionalización, de la concepción de globalización y de empresa, y finalmente desde el entendimiento que de manera "transversal" se tenga de contabilidad. La acción comunicativa de la contabilidad, se genera tras dichas develaciones para construir una sociedad más equitativa e incluyente.

\section{4. ¿Cómo hacer realizable este tipo de trabajos? Algunos apuntes y consideraciones metodológicas que pueden ser observados}

Vinculado con lo descrito hasta ahora, la propuesta de investigación apunta por convertirse en una de tipo cualitativa, interpretativa que se aborda desde posturas críticas de las relaciones internacionales y la contabilidad y de la teoría de la legitimidad y con una orientación a la interpretación de la información que la contabilidad produce en el sentido de la Teoría de la Acción Comunicativa. El desarrollo de la investigación y de la solución a la pregunta central que lo conforma, implica, seguramente, la realización de varias fases. Que se describen y ejemplifican a continuación.

En la primera fase, se recolecta la información financiera, notas a los estados contables y reportes de gestión de empresas "representativas" del modelo económico y de internacionalización, y que serían usadas como estudio de caso.

Aunque lo deseable es que el trabajo tenga el aval de las empresas, ello no es condición insuperable para la realización de la investigación en la medida que se acude a información contable "pública" que se contrasta e interpreta con varios actores económicos como proveedores, trabajadores, usuarios, mesas de tierra, etc.

La información recolectada es analizada en términos no solo cuantitativos (margen de utilidad, impuestos, gastos por salarios, etc.) sino cualitativo identificando las principales explicaciones dadas por la propia empresa para dar cuenta del resultado obtenido. Igualmente para evidenciar los criterios con los que ellas califican sus niveles de responsabilidad social.

Esta información, realizada bajo bases de contabilidad colombianas, se compara con la que debería incluir las empresas al realizar la transición hacía estándares internacionales describiendo los cambios que la nueva regulación genera en términos de la cantidad y tipo de información que debería generar la empresa. 
Una segunda fase recolectaría información directa e indirecta, acerca de la gestión empresarial desde actores sociales y económicos diferentes a la empresa. En este caso, por ejemplo, informes sobre desplazamiento forzado y tierras, violencia sindical, relaciones laborales, condiciones comerciales para la adquisición de bienes y servicios a proveedores, régimen tributario aplicado etc. Permitiría la identificación de determinantes sociales críticas que la propuesta de contabilidad social aquí planteada debería incluir.

Esta información se presentaría como complementaria de los estados, reportes e informes tradicionales y se agrupa como una única y más amplia información contable empresarial, para la trascendencia social.

En la tercera y última fase, la información comprensiva de la gestión social empresarial se presenta a distintos actores sociales y económicos, incluida la empresa, primero de manera "separada" y luego en talleres colectivos, es decir con la participación de los actores sociales identificados. Se trata de construir como el significado social y la interpretación de la gestión empresarial que de la contabilidad social se pueda producir, amplía y modifica cuando existen diálogo, la deliberación social de actores en igualdad de condiciones.

Se pueden complementar estas actividades "de campo" con revisión bibliográfica de textos que aborden el objeto de análisis de la propuesta.

- Interpretación de los estándares internacionales de contabilidad en cuanto a la información que solicitan sea relevada respecto de las partidas que componen los Estados financieros y contables.

- Interpretación de bibliografía pertinente y de documentos oficiales y centros de investigación que den cuenta de los principales problemas sociales del país que sean susceptibles de ser incorporados en la información contable de las organizaciones incluidas en el estudio de caso.

- Entrevistas semi estructuradas con empleados de empresas a estudiar, organizaciones sociales involucradas con la gestión de las em- presas que hacen parte de los estudios de caso, entes gubernamentales, asesores fiscales, $y$ otros actores relevantes.

Finalmente se espera que con el contexto descrito, las opciones de referentes teóricos planteados como alternativas conceptuales para abordar la pregunta central de la investigación propuesta y las opciones metodológicas planteadas se aporten elementos de juicio para ver como la contabilidad y otras disciplinas pueden contribuir a un desarrollo económico para la equidad y la inclusión. El camino apenas está esbozado y sobre los desarrollos y resultados investigativos espero contribuir a la disciplina a la profesión y a la sociedad en general.

\section{Referencias}

Ahumada, C. (2006). El modelo Neoliberal y su impacto en la sociedad Colombiana, El Ancora editores, Bogotá (25-65).

Anderson, P. (1996). Neoliberalismo: un balance provisorio. En: Amir sadel, y Pablo Gentile, Las Trampas del Neoliberalismo. Mercado crisis y exclusión social. Clacso Buenos Aires.

Archel, P, Husillos, J, Larrinaga \& Spence, C. (2009). Social disclosure, Legitimacy Theory and the role of the state. En Accounting, Auditing \& Accountability Journal. Vol 22, 8:1284- 1307.

Arrighi, G. (2007). Adam Smith en Pekin. Orígenes y fundamentos del siglo XXI (traducción de Juanmari Mandariaga) Ediciones Akal. SA Madrid: 166-183.

Barbé, E. (2007). Teoría de las Relaciones Internacionales, en Relaciones Internacionales, Tecnos, Madrid: 41-128.

Beck, U. (2005). La Mirada cosmopolita o la Guerra es la paz? Edición en castellano. Barcelona Paidos.

Bellamy, Jhon. (2007). La financialización del capitalismo. En: 25 años del Neoliberalismo. Hacer Editorial, Barcelona: 41-54.

Bello, Martha. (2006). El desplazamiento forzado en Colombia: acumulación de capital y exclusión social. En: Investigación y desplazamiento forzado, reflexiones éticas y metodológicas. Bogotá Redif y Colciencias: 19-31.

Boron, A. (2008). Socialismos Siglo XXI, ¿̇hay vida después del neoliberalismo? Buenos Aires, Luxemburgo.

Callinicos, A. (1993). Contra el Post modernismo, una crítica marxista. El Ancora Editores, Bogotá: 231 -322.

Castells, M. (1999). La era de la información Volumen I Siglo XXI editores, Madrid: 93-178.

Castillo, N. (2010). La inversión Extranjera en la integración Latinoamericana. En Cuadernos de Estudios Latinoamericanos. No. 7 Instituto de Altos Estudios para el Desarrollo IAED. Bogotá: 97-106.

CESDE, Fundación Centro de Estudios Escuela para el DesarroIlo (2009). Crisis del modelo neoliberal y desigualdad en Colombia: dos décadas de políticas públicas. Ediciones desde abajo: 150-173. 


\section{CONTABILIDAD PARA LA EQUIDAD Y LA INCLUSIÓN SOCIAL: PROPUESTAS PARA UNA INVESTIGACIÓN INTERDISCIPLINARIA \\ A LARGO PLAZO}

Colombia, Congreso Nacional de la República, Ley 1314 de 2009, Julio 13

Consejo Técnico de la Contaduría Pública (2006). Análisis de la implementación de estándares de contabilidad y auditoría en empresas de interés público en Colombia. Divergencias, entre la normativa IASB y la normativa contable colombiana, en Revista Internacional Legis de Contabilidad y Auditoría, No. 27, Julio Septiembre del 2006: 41-52.

De Souza, B. (2003). Los proceso de globalización, en la caída del Angelus Novus: Ensayos para una nueva teoría social y una nueva práctica política. Universidad Nacional de Colombia. Bogotá: 168-242.

De Souza, B. (1999). La globalización del Derecho. Los nuevos caminos de la regulación y la emancipación. Bogotá, Universidad Nacional de Colombia, ILSA editores.

De Souza, B. (2006a). Subjetividad, ciudadanía y emancipación. En: De la Mano de Alicia. Lo social y lo político en la postmodernidad. Siglo del Hombre Editores, Ediciones de la Universidad de los Andes, Bogotá: 285-343.

De Souza, B. (2006 b). Renovar la teoría crítica y reinventar la emancipación social. CLACSO Universidad de Buenos Aires: $9-41$.

Deegan, G. (2006). legitimacy theory. en Methodological Issues in Accounting Research: Theories and Methods. Zahirul Hoque editor general. Espiramus. Australia: 161-181.

Dunne, T. (2007). International Relations Theory. Discipline and diversity. Oxford.

Einchengreen, B. (2000). Hacia Una nueva arquitectura financiera internacional. Instituto de Economía Internacional. Washington: 1-42.

Elias, J. \& Sutch, P. (2007). International Relations. The Basics. New York Routledge.

Estrada, J. (2004). Gestión de la crisis. Reforzamientos de la desregulación económica y "disciplina fiscal" (1999-2004). En: Construcción del Modelo Neoliberal en Colombia 1970. 2004. Ediciones Aurora, Colombia: 65-140.

Evans, P. (2007). Instituciones y desarrollo en la era de la globalización neoliberal. Bogotá, ILSA editores.

Forrester, V. (1997). El Horror Económico. Fondo de Cultura Económica, Buenos Aires: 81-107.

Gago, F. (2005). Administración de la Responsabilidad Social Corporativa. Thompson ed: 1-35.

García Picazo, P. (2004). Las relaciones internacionales en el siglo XX. La contienda teórica. Hacia una visión reflexiva y crítica. 1 ed. Madrid, Universidad Nacional de Educación a distancia.

Giddens, A. (2000). Un mundo desbocado. Madrid, Taurus.

Giraldo, G. (2008). Repercusión de la Teoría Crítica en la Investigación Contable. En: Perspectivas críticas de la contabilidad "reflexiones y críticas contables alternas al pensamiento único" Memorias del VII Simposio Nacional de Investigación Contable y Docencia. CCINCO Universidad Nacional de CoIombia: 77-94.

Gómez M. \& Ospina, C. (2009). Avances interdisciplinarios para una comprensión crítica de la contabilidad. Universidad de Antioquia, Universidad Nacional de Colombia: 13-35.

González, J. (2003). No hay falacia neoliberal en "la Falacia Neo liberal, crítica y perspectivas," Universidad Nacional de Colombia. Bogotá: 85-106.
Gray, R. (2002). The social accounting project and accounting, organizations and Society: privileging engagement, imaginings, new accounting and pragmatism over critieque?. Accounting, Organizations and Society, 27 (7): 687-708.

Griffiths, M. (2007). International Relations Theory for Twenty First Century. An introduction. Routledge.

Harvey, D. (2008). El neo liberalismo como destrucción creativa. En the annals of the american Academy of political and social science 2007, traducción de Germán Legens en www. rebelion,org, agosto de 2008.

Habermas, J. (1985). Ciencias sociales reconstructivas Vs comprensivas. En Conciencia Moral y acción comunicativa, Barcelona Península: 33-55.

Habermas, J. (1990) acciones, actos de habla, interacciones lingüísticamente mediadas y mundo de la vida. En Pensamiento Postmetafísico, Madrid Taurus: 67-107.

Habermas, J. (2002a) Racionalidad del entendimiento. Aclaraciones al concepto de racionalidad comunicativa desde la teoría de los actos del habla. En Verdad y Justificación Madrid, Trotta: 99-131.

Habermas, J. (2002a) Corrección versus verdad. Acerca del sentido de obligación de los juicios y normas morales. En Verdad y Justificación Madrid, Trotta: 99-131.

Habermas, J. (2002b) La teoría de la Racionalización de Max Weber, en Teoría de la Acción Comunicativa I, México, Taurus: 197-350.

Habermas, J. (2006) Tratamiento cultural igualitario y los límites del liberalismo post moderno, en Entre Naturalismo y Religión, Barcelona Paidós: 275-314.

Habermas, J. (2009) Tiene aún la democracia una dimensión epistémica? Investigación empírica y teoría normativa en Ay Europa, Madrid. Trotta: 139-183.

Halabi, Y. (2004). The expansion of Global Governance into the third World: altruism, realism or constructivism. En: International Studies Review, 6: 21-48.

Held, D. \& Mc., Grew, A. (2004). The Global Transformation Reader, Polity, Cambridge: 1-50.

Hobsbawm, E. (1995). Historia del Siglo XX. Barcelona Editorial Crítica: 403-576.

Huntington, S. (1993). The Clash of civilizations. En: Foreign Affairs, Summer 1993, 72 (3): 22-49.

International Accounting Estándar Board, IASB (2010). Conceptual Framework for Financial Reporting 2010. London, UK: 1-67.

Kalmanovitz, S. (2007). Colombia en las dos fases de la globalización. En: Revista Economía Institucional. Universidad Externado de Colombia, 9 (17): 43-75.

Keohane, R. \& NYE, S. (2000). Globalization: Whats new? What's not (and So What). En: Foreing Policy, 118: 104119.

Larrinaga, C. (1999). Perspectivas alternativas de investigación en Contabilidad: una revisión. En: Revista de Contabilidad, 2 (3): 103-131.

Lechner, F. (2008). The globalization Reader, Blackwell Oxford: 217-256.

Luke, M. (2007). The Third wave in globalization Theory, en International Studies Review, 9 (2): 173-196.

Machado, A. (2006). Tenencia de tierras, problema agrario y conflicto. En Investigación y desplazamiento forzado, reflexiones éticas y metodológicas. Bogotá Redif y Colciencias: $81-96$. 
Mantilla, S. (2002). Estándares Internacionales de presentación de reportes financieros. Adiós a las Nic. Un Análisis desde Colombia. En: Guía para la inserción contable colombiana en los Escenarios Internacionales. Serie Soluciones en Desarrollo del IPD Pontificia Universidad Javeriana, Bogotá: 43-82.

Manuzul, A. (2006). Stakeholder theory, en Methodological Issues in Accounting Research: Theories and Methods. Zahirul Hoque editor general. Espiramus. Australia: 207-222.

Martinez, G. (2000). Imperio Transnacional: trasplante y adopción de normas y modelos de contabilidad. En: Revista Contaduría. Universidad de Antioquia, 36.

Messner, D. (1999). La transformación del Estado y la política en el proceso de globalización. En: revista Nueva sociedad, 163: 71-91.

Montilla, O, Montes, C. \& Mejía, E. (2005). Armonización o estandarización contable internacional. Cali, Universidad Libre

Nuñez, J. (2009). Incidencia del gasto público social en la distribución del ingreso, la pobreza y la indigencia". En: Archivos de Economía \#359 Bogotá: Departamento Nacional de Planeación: 2-76.

Pineda, A. (2002). El modelo de propaganda de Noam Chomsky: medios mainstream y control del pensamiento. En: Revista Latina de Comunicación, 47.

Portes, A. (2004). El desarrollo futuro de América Latina. Neoliberalismo, clases sociales y transnacionalismo. Bogotá ILSA editores.

Restrepo, B. \& Dario, I. (2003). De la falacia neoliberal a la nueva política. La Falacia Neoliberal, crítica y perspectivas. Universidad Nacional de Colombia. Bogotá: 17-40.

Reveiz, E. (2004). El desenlace Neoliberal: tragedia o renacimiento. Centro de Investigaciones para el Desarrollo CID de la Universidad Nacional de Colombia. Academia Colombiana de Ciencias Económicas.

Rueda, G. (2010a).El papel de la contabilidad ante la actual realidad económica, social y política del país. Más allá de la convergencia de prácticas mundiales, En: Cuadernos de contabilidad. Pontificia Universidad Javeriana, 28: 76-91.

Rueda, G. (2010b) Estrategias para la inserción empresarial en el mercado global. En: Más allá de la seguridad democrática. Agenda hacia nuevos horizontes. Patrana E. Jost S, Matquez M editores. Editorial Javeriana, KAS, Bogota: 377-398.

Rueda, G. (2011a) Neoliberalismo y convergencia contable. En: Revista Lúmina de la Universidad de Manizales, 11.

Rueda, G. (2011b) Democracia, emancipación social y contabilidad. En: Revista Papel Político Vol 16 No. 1 En proceso de impresión.

Ryan, B, Scapens, R. \& Theobald, M. (2004). Metodología de la investigación en finanzas y contabilidad. Barcelona, ediciones Deusto.

Sierra, F. (2010). Cuestiones epistemológicas de la interdisciplinariedad. Pontificia Universidad Javeriana, Seminario Común, 2010

Stallings, B. (1994). "La influencia internacional en las políticas económicas: deuda, estabilización y reforma estructural". En: la Política de Ajuste Económico: las restricciones internacionales, los conflictos distributivos y el Estado. Cerec Bogotá: 59-116.

Tua, J. (2000). Hacia el triunfo definitivo de las normas internacionales de contabilidad? En: Revista Legis del Contador No. 1, Bogotá: 113-174.

Uribe, C. (2010). Tecnócratas y egresados de universidades estadounidenses: el saber económico, en la construcción neoliberal en Colombia. Ponencia presentada en Seminario Internacional Desarrollo, desigualdades y conflictos sociales. Instituto de Estudios Peruanos.

Wallerstein, I. (1999). La imagen global y las posibilidades alternativas de la evolución del sistema mundo 1945-2025. En: Revista Mexicana de Sociología, 2 (61): 3-34.

Weber, C. (2005). International Relations Theory. $2^{\text {nd }}$ Edition A Critical Introduction. London \& New York. Routledge. 